\title{
O COMPORTAMENTO DO CONSUMIDOR NO MERCADO FITNESS: FÍSICO X VIRTUAL
}

CONSUMER BEHAVIOR IN THE FITNESS MARKET PHYSICAL X VIRTUAL

\section{Mariane Eloisa Mello}

Graduada em Educação Física pela Universidade Feevale (Novo Hamburgo/Brasil).

E-mail: marymello88@gmail.com

\section{Marcelo Curth}

Doutor em Administração pela Universidade do Vale do Rio dos Sinos (São Leopoldo/Brasil).

Professor na Universidade Feevale (Novo Hamburgo/Brasil).

E-mail: marcelocurth@feevale.br

\section{Manuela Albornoz Gonçalves}

Doutora em Administração pela Universidade Federal do Rio Grande do Sul (Porto Alegre/Brasil).

Professora na Universidade Feevale (Novo Hamburgo/Brasil).

E-mail: manu.mag@gmail.com

\section{Rogerio Paulo Joaquim Salvador}

Mestre em Exercício e Saúde pela Universidade de Lisboa (Lisboa/Portugal).

Professor Adjunto do Instituto Politécnico de Leiria (Leiria/Portugal).

E-mail: rogerio.salvador@ipleiria.pt 


\section{RESUMO}

A sobrevivência do profissional de Educação Física, bem como as empresas do mercado fitness, frente a eventos adversos externos, impostos de forma abrupta por uma nova realidade decorrente da pandemia de COVID-19, expôs a fragilidade das relações comerciais com seus alunos/clientes, comprometendo as receitas e forçando-os a se reinventar e adaptar-se às novas modalidades de atendimento. Entendendo que a percepção de valor, confiança, necessidade de interação afetam a intenção de compra do consumidor, este artigo tem como objetivo verificar esta influência no mercado fitness de modo comparativo ao ambiente de entrega do serviço: estabelecimentos com serviços físicos x virtuais. Foi realizado um estudo do tipo survey com 161 alunos/clientes de academias. A técnica estatística utilizada foi Modelagem de Equações Estruturais (MEE), por meio da qual o modelo conceitual foi testado. Os resultados destacam a percepção de que o consumo em lojas físicas obtém uma resposta emocional positiva visando à intenção de compra por parte do consumidor, visto que valoriza bastante a interação humana, gerando sentimentos de alegria, satisfação e bem-estar, sendo sensivel tanto ao preço comportamental como o monetário. Indicam maior percepção de qualidade e confiança na compra de serviços/produtos em ambientes físicos quando comparado à venda no formato virtual, considerando a necessidade de interação do aluno/cliente com o profissional de Educação Física.

Palavras-chave: Percepção de valor. Confiança. Necessidade de interação. Intenção de compra. Fitness.

\section{ABSTRACT}

The survival of Physical Education professionals, as well as companies in the fitness market, faced with adverse external events imposed abruptly by a new reality resulting from the COVID-19 pandemic, exposed the fragility of commercial relations with their students/clients, implicating incomes and forcing them to reinvent themselves and adapt to new ways of costumer services. Therefore, this article aims to identify the influence of the value perception, reability and necessity of interaction in the intention of consumption in the fitness market in physicals spaces $x$ virtuals. A survey-type study was carried out with 161 gym students/clients. The statistical technique used for Structural Equation Modeling (SEM), through which the conceptual model was tested. The results highlight that perception of consumption in physical stores obtains a positive emotional response to the purchase intention on the part of the costumers, whereas it highly values human interaction, generating feelings of joy, satisfaction and well-being, being so sensitive not only with behavioral price as also to monetary. They indicate a greater perception of quality and confidence in the purchase of services/products in special environments when compared to online sales format, considering necessity of interaction between the student/customer and the Physical Education professional.

Keywords: Value perception. Reliability. Necessity of interaction. Purchase intention. Fitness. 


\section{INTRODUÇÃO}

Partindo dos processos desencadeados em 2020, decorrentes da pandemia causada pelo vírus SARS CoV-2 (COVID-19) que assolou o mundo, levando as autoridades sanitárias a elaborarem protocolos com restrições, sendo uma das principais, o isolamento social. Por conta disso, muitas empresas fecharam e/ou suspenderam suas atividades, adotaram o home-office e somente serviços essenciais mantiveram o funcionamento.

As academias de ginástica esvaziaram, tanto por imposição legal, como por opção dos clientes. Contudo, a literatura nos indica que o exercício físico está ligado diretamente à saúde do indivíduo, segundo a Organização Mundial de Saúde (OMS), a não realização de exercícios físicos, pode causar doenças crônicas (WORLD HEALTH ORGANIZATION - WHO, 2011). O estudo de Lee et al. (2012) cita o efeito da atividade/ exercício físico na melhora do sistema imunológico, que é o principal meio de defesa do organismo contra vírus, bactérias e macromoléculas.

Diante deste cenário, tanto os profissionais de Educação Física como as empresas de ginástica (academias de ginástica), foram forçados a encerrar os atendimentos presenciais, levando a uma severa queda de receitas, uma vez que, o aluno/cliente paga pela hora/aula e/ou serviço prestado.

Frente a uma pandemia, empresas e profissionais, visando à recuperação financeira, como também a preocupação com a saúde do aluno/cliente, ampliaram o uso da internet. O mercado fitness (empresas/ profissionais/professores) passaram a comercializar suas aulas de forma on-line. Sendo assim, essa estratégia vai ao encontro do que é mostrado no estudo de Curth et al. (2020) quanto ao crescimento das vendas de e-commerce em todo o mundo nos últimos anos. A necessidade de aproveitamento das ferramentas digitais para se reinventar, dando margem para vendas e atendimentos on-line, um ramo de mercado que está em ascensão. Em contrapartida, o profissional de Educação Física on-line não possui todo o suporte que uma sala de ginástica tem a sua disposição, como por exemplo, equipamentos e outras ferramentas, para o seu aluno/cliente executar os exercícios e movimentos, assim, ele (profissional/ instrutor) precisa ser mais criativo, idealizador de formas de executar seu treino em casa, utilizando o que seu aluno/cliente disponibiliza.

Paradarfrenteao presenteartigo,énecessáriocompreenderasespecificidades dosestabelecimentos, ou seja, lojas físicas e virtuais. Neste sentido, os autores Gonçalves, Costa e Maia (2019) apresentam através dos seus estudos a comparação de compra entre a loja física X virtual, levantando dados relativos de ambas às modalidades. Já no estudo de Garrán (2005), o apontamento é à ascensão do ganho de espaço do comércio eletrônico nas transações comerciais, como também a compra e venda de produtos e serviços por ferramentas digitais. Em Rohden, Matos e Curth (2016) é apresentado o crescimento das 
vendas on-line dando ênfase na intenção de compra do consumidor no mercado virtual, destacando a importância do mercado virtual para a lucratividade de uma empresa.

Mas as perguntas que ficam considerando as lacunas deixadas pela bibliografia, são: Seria este um ramo de negócio que o profissional de Educação Física e as empresas de ginástica deveriam investir? Realmente após a pandemia, será que este tipo de atendimento/venda se consolida, ou é mais uma opção para os alunos/clientes?

Pesquisas em comportamento do consumidor mostram que a intenção de compra do cliente é influenciada por distintas variáveis, tais como, atitudes, fatores individuais, qualidade do serviço, confiança (KIM; FERRIN; RAO, 2008; WEISBERG; TE'ENI; ARMAN, 2011; WELLS; VALACICH; HESS, 2011; PEÑA-GARCÍA, 2020), entre outras. Dessas variáveis, a percepção de valor, confiança e necessidade de interação (fator individual) tem maior probabilidade de variar dado o ambiente de entrega dos serviços fitness e, portanto, exercer distinta influência na intenção de compra.

Partindo do questionamento proposto entre qual o seria uma melhor opção de entrega para o serviço fitness, este artigo tem como objetivo verificar a influência da percepção de valor, confiança, necessidade de interação na intenção de compra do consumidor no mercado fitness de modo comparativo ao ambiente de entrega do serviço: estabelecimentos com serviços físicos $x$ virtuais.

Para tanto, foi realizada uma pesquisa descritiva com estudo do tipo survey com 161 alunos/clientes de academias do mercado da Educação Física na região metropolitana de Porto Alegre e Serra Gaúcha. Para análise, a técnica estatística utilizada foi Modelagem de Equações Estruturais (MEE), por meio da qual o modelo conceitual foi testado.

O artigo apresenta uma revisão da literatura sobre as influências da percepção de valor, confiança e necessidade de interação na intenção de compra. Em seguida, o método utilizado é descrito, detalhando variáveis medidas, descrição da amostra e procedimento utilizado. Os resultados apresentam os principais achados da pesquisa e, por fim, as conclusões também apresentam implicações para a academia e a prática.

\section{FUNDAMENTAÇÃO TEÓRICA}

\subsection{INTENÇÃO DE COMPRA}

Partindo do princípio que a intenção de compra é a junção de comportamentos, é possivel entender através dos estudos de Bagozzi (1983, p. 145) que a "intenção constitui um estado de escolha intencional 
em que alguém realiza uma declaração sobre uma ação futura". Para Taylor e Baker (1994) intenção de compra é o conjunto entre satisfação e qualidade do serviço, bem como também é o objetivo e as expectativas do comprador (DAVIS; BAGOZZI; WARSHAW, 1990), assim como o tipo de produto, seu preço e sua dimensão (MOON; CHADEE; TIKOO, 2008).

Desta forma, ao se analisar o estudo de Eisenbeiss et al. (2014) pode-se observar que a intenção de compra do consumidor está ligada à satisfação, sendo esta a relação entre fornecedores e compradores. Isso tem encontro direto no agregado da satisfação, não somente em satisfação específica no ato de comprar, mas sim no ato de querer mais tarde realizar outra compra. Devido a isto, empresas partem do princípio de aumentar seu entrosamento com o consumidor, gerando valor afetivo.

Como mostra o estudo de Oliver (2010) satisfação é um complemento de prazer, o que significa uma ferramenta de consumo para suas necessidades, vontades e desejos. Assim o autor reforça que, o cliente ao se sentir satisfeito em realizar uma compra e tem suas necessidades preenchidas, acaba gerando o desejo de recomprar o serviço prestado ou produto adquirido, tornando-se consumidor fiel.

Kim, Ferrin e Rao (2008), mostram uma vantagem importante que pode influenciar a intenção de compra do consumidor e diz respeito às gratificações e reparações associados a ofertas que transmitem algum valor perceptível. Sendo assim, quanto maior for à assimilação referente ao valor, maior será também a intenção de compra, logo quanto maior for a percepção de valor, maior será a intenção de compra

Já Wells, Valacich e Hess (2011), apontam que intenção de compra está ligada diretamente a um estágio mental, como por exemplo, a tomada de decisão do consumidor, visto que, o mesmo se projeta a adquirir algo, produto ou serviço, ele produz um sinal, uma tomada de decisão de compra.

Em outra ótica, Farias et al. (2014) trazem uma visão de intenção de compra do consumidor fazendo referência a dois fatores: a compra por fatores hedônicos (prazer, diversão ou satisfação) ou a compra por fatores de utilidade (finalidade laboral ou fatores racionais) sendo este, com finalidade na utilização do produto/serviço no trabalho, estudo ou atividade de necessidade básica.

Além da influência de percepção de valor, satisfação do consumidores e qualidade dos serviços, a intenção de compra é influenciada por outras variáveis, tais como atitudes, crenças, fatores individuais (PEÑA-GARCÍA, 2020), preferências de marca (HELLIER, 2003), confiança do consumidor (WEISBERG; TE'ENI; ARMAN, 2011). Dentre estas, a confiança e o fator individual de necessidade de interação são de interesse nesse estudo, pois são variáveis que são influenciadas ou percebidas de forma distinta pelo consumidor dado o ambiente de entrega dos serviços (ambiente físico versus on-line), já, por exemplo, preferência de marca não foi analisada, visto que é constante entre os diferentes ambientes de entrega 
dos serviços. A seguir as variáveis analisadas no estudo e a proposta influência na intenção de compra são detalhadas.

\subsection{PERCEPÇÃO DE VALOR}

Ao decorrer dos tempos, vários são os modelos de valores percebidos em produtos/serviços, assim como também suas dimensões. No estudo de Zeithaml (1988), é trazida a combinação entre qualidade, preço pago pelo produto/serviço e intuição individual (subjetiva) na qual o consumidor se refere ao valor do produto/serviço. Alguns anos mais tarde, é possivel ver no estudo de Zeithaml e Bitner (2003) que a percepção de valor não está apenas no produto em si, mas sim, no valor percebido em toda a bagagem que envolve a compra e o consumo.

Sendo assim, é possível analisar duas abordagens teóricas diferentes no que se diz respeito à percepção de valor, levando em consideração aos estudos citados anteriormente, onde Zeithaml (1988) tem uma perspectiva unidimensional ou global, que está associada ao valor percebido referente ao vínculo (concreto e não concreto) de vantagens e dificuldades da compra, onde se conclui que, o comprador busca ampliar a utilidade nesse processo. Já em outros estudos, como demonstra o Quadro 1, é possível verificar uma perspectiva multidimensional ou analítica, que o valor percebido se refere ao resultado de dimensões inter-relacionadas ressaltando os critérios social, emocional ou funcional, reconhecidos pelo consumidor, acreditando assim que, a empresa que obtiver a melhor entrega de tais fatores/critérios retém uma vantagem competitiva. 


\section{Gestãoe \\ Desenvolvimento}

Quadro 1 - Abordagens multidimensionais do valor percebido

\begin{tabular}{|c|c|}
\hline Autor & Dimensão \\
\hline Sheth, Newman e Gross (1991) & $\begin{array}{l}\text { Valor social } \\
\text { Valor emocional } \\
\text { Valor funcional } \\
\text { Valor epistêmico } \\
\text { Valor condicional }\end{array}$ \\
\hline Groth (1995) & $\begin{array}{l}\text { Cognitiva: utilidade percebida } \\
\text { Psicológica } \\
\text { Interna } \\
\text { Externa }\end{array}$ \\
\hline Grönroos et al. (2000) & $\begin{array}{c}\text { Cognitiva } \\
\text { Emocional (psicológica) }\end{array}$ \\
\hline De Ruyter et al. (1997) & $\begin{array}{c}\text { Dimensão emocional ou valor intrínseco } \\
\text { Dimensão funcional ou valor extrínseco } \\
\text { Dimensão lógica }\end{array}$ \\
\hline Sweeney, Soutar e Johnson (1999) & $\begin{array}{l}\text { Valor social (aceitabilidade) } \\
\text { Valor emocional } \\
\text { Valor funcional (preço/valor pelo dinheiro) } \\
\text { Valor funcional (desempenho/qualidade) } \\
\text { Valor funcional (versatilidade) }\end{array}$ \\
\hline Sweeney e Soutar (2001) & $\begin{array}{l}\text { Dimensão funcional (econômica e qualidade) } \\
\text { Dimensão social } \\
\text { Dimensão emocional }\end{array}$ \\
\hline Petrick (2002) & $\begin{array}{l}\text { Preço comportamental } \\
\text { Preço monetário } \\
\text { Resposta emocional } \\
\text { Qualidade } \\
\text { Reputação }\end{array}$ \\
\hline
\end{tabular}

Fonte: Adaptado de Sánchez et al. (2006).

Considerando as dimensões apresentadas no Quadro 1 e partindo da importância das variáveis da percepção de valor abordadas por Petrick (2002), que de forma resumida são:

a. qualidade (Qual): essa dimensão relaciona-se às especificações, a harmonização ao uso e a um conjunto de fatores que agradam (ou não) o consumidor, como o atendimento, por exemplo. Tem relação direta com a confiança no prestador de serviço;

b. resposta emocional (Re): como o bem/serviço faz um consumidor se sentir. É um julgamento, uma sensação de alegria e encantamento que emerge (ou não) da relação com o prestador de serviços; 
c. preço comportamental (Pc): está relacionado ao desgaste para adquirir um bem/serviço. Envolve o tempo consumido e o esforço emocional para isso;

d. preço monetário (Pm): é o montante de dinheiro gasto, uma relação do custo financeiro com o que se recebe em troca;

e. reputação (Rep): tem relação com a imagem e o prestígio do prestador de serviço perante o mercado. Se são organizações respeitadas e/ou que apresentam status.

Os estabelecimentos físicos do mercado fitness entregam ao consumidor um grau de tangibilidade maior (em média) que os estabelecimentos em ambientes on-line, isso afeta as dimensões das variáveis de percepção de valor propostas por Petrick (2002) e as suas relações possíveis com a comportamento de consumo, mais especificamente com a intenção de compra, portanto, sugere-se as seguintes hipóteses $(\mathrm{H})$ :

H1: A dimensão qualidade (a), resposta emocional (b), preço comportamental (c), preço monetário (d) e reputação (e) influencia positivamente a intenção de compra de serviços no mercado fitness com estabelecimento FísıCO quando comparado aos serviços do mercado fitness VIRTUAL.

\subsection{CONFIANÇA}

Como definição para a palavra confiança, a literatura sugere um conceito multidimensional (MCCOLE; RAMSEY; WILLIAMS, 2010). A confiança é um sentimento do indivíduo/consumidor na totalidade de um determinado atendimento ou serviço, de acordo com a lealdade do cumprimento das promessas do vendedor (EISINGERICH; BELL, 2008).

Confiança para Ba e Pavlou (2002) seria a troca que ocorre de maneira compacta de expectativas de confiança relacionadas ao indivíduo. Já no que diz respeito a compras on-line, confiança é a crença de confiabilidade, relacionado ao entendimento de competência, integridade, benignidade (MCKNIGHT; CHOUDHURY; KACMAR, 2002).

Deliberali, Brandão e Bizarrias (2019) destacam que a confiança não está ligada a valores hedônicos, tendo em vista que, este valor está mais ligado a condições de vida. Mas é possível medir sua confiança, uma vez que, consumidores com baixa confiança apresentam menor satisfação mesmo havendo valores hedônicos. Porém, ao aumentar sua confiança, automaticamente seu nível de satisfação também se eleva, e essa confiança gera valor hedônico onde aumenta a satisfação.

Levando em consideração o nivel de confiança, sendo que muitas vezes leva a fidelização, uma boa forma de conquistar a confiança do cliente é no momento da assistência do serviço, com esclarecimento de dúvidas de forma simples e direta, diminuindo as inseguranças e mostrando todo o suporte existente 
quanto às dificuldades ou dúvidas que venham a surgir do cliente. Dessa maneira potencializa o vínculo entre empresa e cliente. Levando em consideração a área da Educação Física, base desta pesquisa, compreende-se que o exercício físico gera emoção, vínculo e interesse em comum entre os indivíduos.

Além disso, é possivel afirmar que confiança é fundamental no relacionamento com o cliente, principalmente no ambiente virtual, sabendo das peculiaridades que existem neste tipo de negociações (CHEN; BARNES, 2007; MCCOLE; RAMSEY; WILLIAMS, 2010) e que em alguns formatos de interação isso pode ser influenciado, sendo o caso das interações virtuais.

Conforme Terzia (2011) o e-commerce(loja virtual) tornou-se uma importante ferramenta/plataforma de aproximação entre empresa e consumidor. De acordo com Wilson e Abel (2002) e-commerce tem por definição a realização de negócios através do uso de mídias eletrônicas.

Outro ponto importante que deve ser levado em consideração quando se refere à confiança na compra, principalmente em compras on-line, é a proteção do consumidor (CURTH et al. 2020). São pontos relevantes neste sentido, a segurança nas transações, a facilidade do uso de website, assim como também a privacidade dos dados pessoais (SCHAUPP; BÉLANGER, 2005).

Visando a construção de um relacionamento de confiança entre o indivíduo/consumidor e os prestadores de serviços/comércio eletrônico, Miyazaki e Fernandez (2001), McCole, Ramsey e Williams (2010) destacam que quanto maior a utilização da internet, menor se torna as preocupações referentes à privacidade, o que pode vir a ser algo positivo, tendo em vista a decisão de compra.

De acordo com Deheshti, Firouzjah e Alimohammadi (2016) para criar uma relação de confiança com o consumidor é necessário haver uma boa experiência dentro das organizações esportivas comerciais e profissionais, estabelecendo assim a lealdade e frequência de consumo dos clientes.

Assim, entendendo haver uma possivel relação entre a confiança na compra de serviços no mercado fitness, considerando a comparação entre o físico e o virtual, sugere-se a seguinte hipótese:

H2: A confiança influencia positivamente na intenção de compra de serviços no mercado fitness com estabelecimento FísıCO quando comparado aos serviços do mercado fitness VIRTUAL.

\subsection{NECESSIDADE DE INTERAÇÃO}

Conforme Steenkamp e Maydeu-Olivares (2015), a necessidade de interação é uma peculiaridade do consumidor, que consiste em pensamentos, sentimentos e comportamentos da pessoa na atividade do consumidor. Desta forma, a interação torna-se uma particularidade favorável do consumidor/cliente que deve ser investido (COLLIER; KIMES, 2013; LEE, 2017).

Segundo Baumeister e Leary (1995) a necessidade de interagir do indivíduo se deve a um sentimento de ansiedade, sendo esse um motivo interpessoal fundamental, que explica de forma significativa 
o comportamento humano. Assim, Rubin, Perse e Powell (1985) afirmam que o comportamento do consumidor varia de acordo com a interação social e a forma que demonstram satisfação com a mesma.

Observando a loja física, a interação acontece o tempo inteiro, uma vez que os vendedores/ atendentes, servem como uma forma de ligação entre o produto/serviço e o consumidor. São conhecidos também como "facilitadores-chave" potencializando a venda direta visando a satisfação do cliente (GOUDGE et al., 2017). Assim, Moreira (2007) complementa que ao entender o desejo do cliente/consumidor e ser especialista em um ramo é uma vantagem para a satisfação e confiança.

Mesmo com os avanços da internet, muitos consumidores ainda preferem o contato humano e, neste sentido, Moreira (2007) ressalta a importância dos vendedores, que tem por obrigação levar um serviço completo para todos os clientes/consumidores que entram na empresa, desde uma boa assistência até a finalização da compra.

$\mathrm{Na}$ era da Tecnologia do Autoatendimento (TAA), muitos consumidores preferem essa "independência", o qual não necessita do envolvimento direto de uma pessoa/prestador de serviço (MEUTER et al., 2000). Desta forma, as empresas investem em TAA visando a sua eficiência (WALKER et al., 2002), elevando o grau de satisfação do seu cliente/consumidor disponibilizando mais uma ferramenta como canal de serviço (DEMIRCI OREL; KARA, 2014; MEUTER et al., 2003; RUST; ESPINOZA, 2006).

Levando em consideração o grau de importância da necessidade de interação no serviço disponibilizado no mercado fitness, como por exemplo, treinos personalizados e a influência que pode ocorrer na compra em loja física e on-line, sugere-se a seguinte hipótese:

H3: A necessidade de interação influencia positivamente na intenção de compra em serviços no mercado fitness com estabelecimento FísıCO quando comparado aos serviços no mercado fitness VIRTUAL.

\section{METODOLOGIA}

Considerando o objetivo descritivo proposto na pesquisa, foi realizado um estudo do tipo survey. $O$ instrumento de pesquisa contou com a seleção de escalas que foram utilizadas e validadas em outros estudos e contou com 31 questões, sendo 25 sobre os construtos da pesquisa, utilizando a escala de Likert de 5 pontos, sendo 1 "Discordo totalmente" e 5 "Concordo totalmente".

O questionário foi aplicado de forma on-line, levando em consideração a necessidade de isolamento social, devido ao COVID-19, sendo o método mais seguro e eficaz para o momento. 
As questões sobre o construto Qualidade foram adaptadas de Petrick (2002), ressaltando o conjunto de fatores que agradam (ou não) o consumidor, como no caso do atendimento que tem relação direta com a confiança no prestador de serviço: QUAL1: os serviços do mercado fitness com estabelecimento FísICO possuem qualidade excepcional, quando comparadas aos serviços do mercado fitness VIRTUAL (ON-LINE); QUAL2: os serviços do mercado fitness com estabelecimento FíSICO possuem maior atenção no atendimento, quando comparadas aos serviços do mercado fitness VIRTUAL (ON-LINE); QUAL3: os serviços do mercado fitness com estabelecimento FísICO possuem atendimento melhor, quando comparadas aos serviços do mercado fitness VIRTUAL (ON-LINE).

O construto Resposta Emocional, também foi adaptado de Petrick (2002), levando em consideração como o bem/serviço faz o consumidor se sentir, uma sensação de alegria, encantamento (ou não) da relação com o prestador de serviço: Re1: os serviços do mercado fitness com estabelecimento Físıco fazem eu me sentir melhor, quando comparadas aos serviços do mercado fitness VIRTUAL (ON-LINE); Re2: os serviços do mercado fitness com estabelecimento FíSICO me dão sensação de alegria na transação, quando comparadas aos serviços do mercado fitness VIRTUAL (ON-LINE); Re3: os serviços do mercado fitness com estabelecimento FísıCO fazem eu me sentir satisfeito, quando comparadas aos serviços do mercado fitness VIRTUAL (ON-LINE).

Seguindo a mesma linha, o construto relacionado a Preço Monetário, também foi adaptado de Petrick (2002), considerando uma relação de custo financeiro com o que se recebe em troca, dinheiro gasto: Pm1: os serviços do mercado fitness com estabelecimento FísICO apresentam uma boa relação custo-benefício, quando comparadas aos serviços do mercado fitness VIRTUAL (ON-LINE); Pm2: os serviços do mercado fitness com estabelecimento FísICO valem o custo financeiro investido, quando comparadas aos serviços do mercado fitness VIRTUAL (ON-LINE); Pm3: os serviços do mercado fitness com estabelecimento FÍSICO possuem preços justos, quando comparadas aos serviços do mercado fitness VIRTUAL (ON-LINE); Pm4: os serviços do mercado fitness com estabelecimento FísICO são econômicos, quando comparadas aos serviços do mercado fitness VIRTUAL (ON-LINE).

O construto Preço Comportamental, partiu do estudo de Petrick (2002), que está relacionado ao desgaste para adquirir um bem/serviço, levando em consideração o tempo consumido e o esforço mental para tanto: Pc1: os serviços do mercado fitness com estabelecimento FísıCO são fáceis de serem contratados, quando comparados aos serviços do mercado fitness VIRTUAL (ON-LINE); Pc2: os serviços do mercado fitness com estabelecimento FíSICO requerem pouco esforço (energia) para serem contratados, quando comparados aos serviços do mercado fitness VIRTUAL (ON-LINE); Pc3: os serviços do mercado fitness com estabelecimento FíSICO são mais facilmente comprados, quando comparados aos serviços do mercado fitness VIRTUAL (ON-LINE). 
Da mesma forma, o construto Reputação, está baseado em Petrick (2002) onde é a relação com a imagem e o prestígio do prestador de serviço diante do mercado, se é uma organização respeitada, apresenta status: Rep1: os serviços do mercado fitness com estabelecimento FísICO possuem boa reputação, quando comparados aos serviços do mercado fitness VIRTUAL (ON-LINE); Rep2: os serviços do mercado fitness com estabelecimento FíSICO são bastante respeitados, quando comparados aos serviços do mercado fitness VIRTUAL (ON-LINE); Rep3: os serviços do mercado fitness com estabelecimento FÍSICO apresentam bom status, quando comparados aos serviços do mercado fitness VIRTUAL (ON-LINE).

Já o construto de Confiança, foi adaptado dos autores McCole, Ramsey e Williams (2010) assim como também dos autores Curth et al. (2020) levando em consideração o grau de confiança, proteção e segurança do consumidor com relação ao serviço: Conf1: eu sinto que minha privacidade está mais protegida quando realizo compras de serviços do mercado fitness com estabelecimento FísICO, quando comparado aos serviços do mercado fitness VIRTUAL (ON-LINE); Conf2: eu me sinto mais seguro quando realizo compras de serviços do mercado fitness com estabelecimento FÍSICO, quando comparado aos serviços do mercado fitness VIRTUAL (ON-LINE); Conf3: eu sinto que os serviços do mercado fitness com estabelecimento FÍSICO tem mais características necessárias para segurança (assistência, personalização, recursos de materiais, por exemplo), quando comparado aos serviços do mercado fitness VIRTUAL (ONLINE).

Agora o construto Intenção de Compra, foi adaptado dos autores Eisenbeiss et al. (2014) e Oliver (2010) destacando o grau de satisfação que o indivíduo demonstra na intenção de realizar a compra do produto/serviço: Inter1: na próxima vez que for comprar, a probabilidade de eu escolher serviços do mercado fitness com estabelecimento FísıCO é maior, quando comparado aos serviços do mercado fitness VIRTUAL (ON-LINE); Inter2: a probabilidade de fazer novas compras do mesmo serviço do mercado fitness com estabelecimento FíSICO é maior, quando comparado aos serviços do mercado fitness VIRTUAL (ONLINE); Inter3: a probabilidade de compras do novos serviços no mercado fitness com estabelecimento FísICO é maior, quando comparado aos serviços do mercado fitness VIRTUAL (ON-LINE).

E por fim, o construto de Interação do consumidor, foi adaptado dos autores Goudge et al. (2017) e Moreira (2007) destacando o grau de importância da interação com o consumidor visando a necessidade do contato humano: Int1: o contato humano durante a prestação de serviços do mercado fitness com estabelecimento FíSICO, torna o processo mais seguro para o consumidor, quando comparados ao serviço do mercado fitness VIRTUAL (ON-LINE); Int2: eu gosto de interagir com a pessoa que presta o serviço para mim no serviço do mercado fitness com estabelecimento FíSICO, quando comparados aos serviços do mercado fitness VIRTUAL (ON-LINE); Int3: acho desconfortável executar exercícios de frente para um celular; Int4: o uso de ambientes virtuais me deixa ansioso/ansiosa. 
E as últimas seis questões são sobre o perfil dos respondentes (sexo, a faixa etária, cidade que reside, renda bruta mensal, grau de escolaridade e ciclo de vida familiar).

A aplicação do pré-teste ocorreu com 19 respondentes e foi realizado no mês de março de 2021 e teve como objetivo a análise de confiabilidade da escala utilizada, considerando os valores do Alpha de Cronbach. Segundo Hair Junior et al. (2009), os valores de Alpha devem estar acima de 0,7.

Os resultados apresentaram valores satisfatórios, tendo a Qualidade um Alpha de 0,74 e 3 indicadores; Resposta Emocional contou com Alpha de 0,93 e 3 indicadores; Preço Monetário apresentou - Alpha de 0,82 e 4 indicadores; Preço Comportamental contou com o Alpha 0,79 e 3 indicadores; Reputação apresentou Alpha de 0,77 e 3 indicadores; Confiança apresentou Alpha de 0,87 e 3 indicadores; Necessidade de Interação apresentou Alpha 0,75 e 3 indicadores e, por fim, Intenção de compra o Alpha de 0,83 e 3 indicadores. Por fim, os resultados apresentados demonstraram que os valores de Alpha estavam de acordo com o sugerido pela literatura, sugerindo a continuidade das etapas sequentes.

$\mathrm{O}$ instrumento de pesquisa foi aplicado de março de 2021 a maio de 2021. A pesquisa contou com o total de amostras de 161 respondentes válidos (usuários de serviços de academias). Considerando o número da amostra, Hair Junior et al. (2009) sugerem que devam ser considerados os parâmetros analisados, sendo entre 5 a 10 respondentes, sugerindo o alinhamento da amostra coletada e os parâmetros utilizados nesta pesquisa.

Para a operacionalização da análise dos dados, foi utilizado o software Microsoft Excel ${ }^{\circledR} 2010$ e SPSS 24 (Statistical Package for Social Sciences), para os dados descritivos e preparação de dados. O software AMOS тм 20 (Analysis of Moment Structures) foi utilizado para a modelagem de equações estruturais (MEE) por ser o mais adequado para tal tipo de análise (GARSON, 2012).

O perfil dos participantes apresentou que, dentre seus 161 respondentes, $115(71,42 \%)$ eram do sexo feminino e na sua maioria na faixa etária entre 26 e 30 anos, somando 45 (27,95\%) respondentes. Quanto a renda bruta, dois grupos tiveram destaque, sendo o da renda bruta de até $R \$ 1.908,00$ (53 $32,9 \%$ ) e entre $R \$ 2.862,01$ e $R \$ 5.724,00(51$ - 31,7\%) e com graduação incompleta $(77-47,8 \%)$. Com relação ao ciclo de vida familiar, os resultados chamaram a atenção pela considerada heterogeneidade, pois os respondentes em relacionamentos e com filhos apresentaram maior percentual (64 - 39,75\%), seguido de em relacionamento e sem filhos $(50-31,1 \%)$ e solteiro sem filhos $(36-22,4 \%)$. 


\section{RESULTADOS E ANÁLISE}

\subsection{ANÁLISE FATORIAL EXPLORATÓRIA (AFE)}

Antes da aplicação de medida do modelo, foi realizada a AFE para a identificação do alinhamento entre os fatores que estão sendo propostos para este estudo (HAIR et al. 2009). Para a operacionalização foi utilizado o software estatístico SPSS 24, sendo realizada a extração dos fatores por meio da análise de componente e principal (ACP). Todas as variáveis apresentaram as comunalidades maiores que 0,50 (Tabela1).

Tabela 1 - Comunalidades

\begin{tabular}{cccccc}
\hline Itens & Inicial & Extração & Itens & Inicial & Extração \\
\hline Qual1 & 1.000 & 0,570 & Rep1 & 1.000 & 0,737 \\
Qual2 & 1.000 & 0,811 & Rep2 & 1.000 & 0,871 \\
Qual3 & 1.000 & 0,778 & Rep3 & 1.000 & 0,795 \\
Re1 & 1.000 & 0,739 & Conf1 & 1.000 & 0,718 \\
Re2 & 1.000 & 0,713 & Conf2 & 1.000 & 0,754 \\
Re3 & 1.000 & 0,737 & Conf3 & 1.000 & 0,656 \\
Prm1 & 1.000 & 0,669 & IR1 & 1.000 & 0,734 \\
Prm2 & 1.000 & 0,723 & IR2 & 1.000 & 0,737 \\
Prm3 & 1.000 & 0,713 & IR3 & 1.000 & 0,674 \\
Prm4 & 1.000 & 0,600 & Inter1 & 1.000 & 0,699 \\
Prc1 & 1.000 & 0,644 & Inter2 & 1.000 & 0,759 \\
Prc2 & 1.000 & 0,638 & Inter3 & 1.000 & 0,808 \\
Prc3 & 1.000 & 0,632 & Inter4 & 1.000 & 0,510 \\
\hline
\end{tabular}

Fonte: Elaborado pelos autores

Antes da etapa de extração final dos fatores, foi realizado o teste de esfericidade de Bartlett $(3293,972)$ e a medida de Kaiser-Meyer-Olkin (KMO) $(0,915)$ para verificar a adequação da amostragem, demonstrando correlação significativa entre as variáveis. Após, todos os fatores foram inseridos para o teste de Eigenvalue com valores acima de 1 . 0 resultado dos seis fatores apresentou 69,806 por cento da variância total explicada (Tabela 2).

Tabela 2 - Variância Total Explicada

\begin{tabular}{cccccccccc}
\hline & \multicolumn{3}{c}{ Initial Eigenvalue } & \multicolumn{3}{c}{ Extraction Sums of Squared Loading } & \multicolumn{2}{c}{ Rotation Sums of Squared Loading } \\
\hline Comp & Total & \% Variância \% Cumulativa & Total & \% Variância & \% Cumulativa & Total & \% Variância & \% Cumulativa \\
\hline $\mathbf{1}$ & 11,28 & 43,409 & $\mathbf{4 3 , 4 0 9}$ & 11,28 & 43,409 & $\mathbf{4 3 , 4 0 9}$ & 4,349 & 16,728 & 16,728 \\
$\mathbf{2}$ & 1,83 & 7,069 & $\mathbf{5 0 , 4 7 7}$ & 1,83 & 7,069 & $\mathbf{5 0 , 4 7 7}$ & 3,517 & 13,528 & 30,257 \\
$\mathbf{3}$ & 1,53 & 5.916 & $\mathbf{5 6 , 3 9 3}$ & 1,53 & 5.916 & $\mathbf{5 6 , 3 9 3}$ & 2,991 & 11,502 & 41,759 \\
$\mathbf{4}$ & 1,269 & 4,882 & $\mathbf{6 1 , 2 7 5}$ & 1,269 & 4,882 & $\mathbf{6 1 , 2 7 5}$ & 2,689 & 10,343 & 52,102 \\
$\mathbf{5}$ & 1,173 & 4,511 & $\mathbf{6 5 , 7 8 6}$ & 1,173 & 4,511 & $\mathbf{6 5 , 7 8 6}$ & 2,559 & 9,844 & 61,945 \\
$\mathbf{6}$ & 1,045 & 4,020 & $\mathbf{6 9 , 8 0 6}$ & 1,045 & 4,020 & $\mathbf{6 9 , 8 0 6}$ & 2,044 & 7,860 & 69,806 \\
\hline
\end{tabular}

Fonte: Elaborado pelos autores 


\subsection{PROPRIEDADE DAS MEDIDAS}

Os indicadores de desempenho do modelo conceitual apresentam a diferença por graus de liberdade 2,86, com significância menor do que 0,05, indicando boa base para qualidade dos outros índices. O GFI, que serve para identificar o ajuste do modelo, apresentou valor de 0,87 , indicando bom ajuste, pois o valor está próximo de 0,90. 0 valor de RMSEA, que é um índice de parcimônia que tenta corrigir as falhas de medida, apresentou-se igual a 0,085, o que indica o ajuste do modelo com a população pesquisada. Os demais índices apresentaram cargas dentro do sugerido pela literatura (HAIR et al. 2009), reforçando a adequação do modelo.

Considerando a etapa de análise estatística dos dados, foram utilizados procedimentos estatísticos consolidados para a realização do tratamento dos dados, análise de confiabilidade e validade das questões. Ao final do processo de análise, foi utilizada a modelagem de equações estruturais para a realização das análises estatísticas, verificação do modelo conceitual e teste de mediação de variáveis.

Após a análise e retiradas de outliers, na qual não foram realizadas exclusões de respondentes, foram verificadas análises de confiabilidade composta (CC) e validade das medidas, sendo realizada pela análise da variância extraída (AVE), como mostra a Tabela 1.

\begin{tabular}{|c|c|c|c|c|c|c|c|c|}
\hline \multirow[t]{2}{*}{ Constructo } & \multicolumn{4}{|c|}{ Modelo de Medida Inicial } & \multicolumn{4}{|c|}{ Modelo de Medida Purificado } \\
\hline & $\begin{array}{l}\mathrm{N}^{\circ} \text { de } \\
\text { itens }\end{array}$ & $\begin{array}{l}\text { Alpha de } \\
\text { Cronbach }\end{array}$ & CC & AVE & $\begin{array}{l}N^{0} \text { de } \\
\text { itens }\end{array}$ & $\begin{array}{l}\text { Alpha de } \\
\text { Cronbach }\end{array}$ & CC & AVE \\
\hline Qual & 3 & 0,79 & 0,85 & 0,75 & - & - & - & - \\
\hline $\operatorname{Re}$ & 3 & 0,89 & 0,89 & 0,76 & - & - & - & - \\
\hline $\mathrm{Pm}$ & 4 & 0,86 & 0,88 & 0,69 & - & - & - & - \\
\hline Pc & 3 & 0,81 & 0,82 & 0,65 & - & - & - & - \\
\hline Rep & 3 & 0,88 & 0,86 & 0,75 & - & - & - & - \\
\hline Conf & 3 & 0,88 & 0,88 & 0,73 & - & - & - & - \\
\hline Inter & 4 & 0,57 & 0,72 & 0,58 & 3 & 0,75 & 0,85 & 0,72 \\
\hline Int & 3 & 0,83 & 0,86 & 0,81 & - & - & - & - \\
\hline Índices sugeridos & & $>0,70$ & $>0,70$ & $>0,50$ & & $>0,70$ & $>0,70$ & $>0,50$ \\
\hline
\end{tabular}

Os indicadores dos construtos apresentaram cargas dentro do sugerido, com exceção do construto Inter que apresentou carga de Alpha de Cronbach de 0,33 (>0,70). Por conta disso, foi realizada a exclusão 


\section{Gestãoe \\ Desenvolvimento}

do indicador Inter4 do modelo e realizada novamente a análise, o que possibilitou a adequação do construto.

Após a purificação dos dados apresentados na Tabela 1, outro teste de validade discriminante foi aplicado. Seguindo a interpretação, os valores de $\mathrm{R}^{2}$ devem ser menores do que os valores de AVE (Tabela 2), não havendo a necessidade de testes posteriores, considerando os valores encontrados.

Tabela 2 - Teste de validade discriminante

\begin{tabular}{ccccccccc}
\hline Constructo & Qual & Re & Pm & Pc & Rep & Conf & Inter & Int \\
\hline Qual & $\mathbf{0 , 7 5}$ & & & & & & \\
Re & 0,45 & $\mathbf{0 , 7 6}$ & & & & & \\
Pm & 0,30 & 0,58 & $\mathbf{0 , 6 9}$ & & & & \\
Pc & 0,33 & 0,42 & 0,52 & $\mathbf{0 , 6 5}$ & & & \\
Rep & 0,45 & 0,37 & 0,35 & 0,35 & $\mathbf{0 , 7 5}$ & & \\
Conf & 0,38 & 0,45 & 0,35 & 0,27 & 0,38 & $\mathbf{0 , 7 3}$ & & \\
Inter & 0,45 & 0,60 & 0,48 & 0,40 & 0,31 & 0,50 & $\mathbf{0 , 7 2}$ & \\
Int & 0,34 & 0,56 & 0,59 & 0,48 & 0,37 & 0,51 & 0,49 & $\mathbf{0 , 8 1}$ \\
\hline
\end{tabular}

Fonte: Elaborada pelos autores

\subsection{ANÁLISE DE DADOS E DISCUSSÃO}

Na Tabela 3 são apresentados os coeficientes padronizados e respectivo nível de significância, sendo possível observar que todas as hipóteses foram suportadas.

Tabela 3 - Cargas dos construtos do modelo conceitual

\begin{tabular}{ccccc}
\hline \multirow{2}{*}{ Hipóteses } & \multicolumn{3}{c}{ Valores } \\
\cline { 3 - 5 } & Coeficientes padronizados & Sig & Suporte para hipótese \\
\hline H1a & Qual - Int & 0,220 & 0,030 & Suportada \\
H1b & Re - Int & 0,250 & 0,025 & Suportada \\
H1c & Pc - Int & 0,290 & 0,027 & Suportada \\
H1d & Pm - Int & 0,550 & 0,001 & Suportada \\
H1e & Rep - Int & 0,220 & 0,030 & Suportada \\
H2 & Conf - Int & 0,450 & 0,020 & Suportada \\
H3 & Inter - Int & 0,600 & 0,001 & Suportada \\
\hline & & Nota: Sig = Significância (p<0,05).
\end{tabular}

A hipótese H1a tratou da relação entre qualidade e a intenção de compra em academias de ginástica com serviço no formato FíSICO quando comparada às academias com serviços no formato VIRTUAL. A 
qualidade apresentou um efeito positivo na intenção de compra nas academias físicas, com valores de $\beta=0,220$ e significância 0,03 ( $p<0,05)$, suportando a hipótese. 0 resultado sugere que a qualidade no ambiente físico é um elemento presente principalmente quando está associado ao atendimento, uma vez que, os alunos/clientes demonstram a necessidade de interação com o profissional de Educação Física. No ambiente físico é possível corrigir movimentos, ter auxílio de equipamentos diversos, para execução e aperfeiçoamento dos treinos, o que gera no consumidor uma maior sensação de qualidade.

A relação da hipótese $\mathrm{H} 1 \mathrm{~b}$ abordou a relação entre a resposta emocional e a intenção de compra em academias de ginástica no formato FísICO quando comparada às academias com serviço no formato VIRTUAL. A resposta emocional para o formato físico, comparada com o virtual, apresentou um efeito positivo na intenção de compra, com valores de $\beta=0,250$ e significância 0,025 ( $p<0,05)$, suportando a hipótese. O resultado demonstra que o ambiente físico transmite maior sensação de alegria, encantamento e engajamento do consumidor com o produto/serviço, isso pode ser alcançado pelo contato direto do profissional de Educação Física que extrapola meramente a execução do exercício e a sua supervisão, construindo uma relação afetiva e de parceria, o que resulta na maior probabilidade de recompra (PETRICK, 2002).

Na relação entre as dimensões de preço (comportamental) H1c e (monetário) H1d e a intenção de compra em academias de ginástica com o formato FíSICO quando comparada às VIRTUAIS, ambas tiveram suporte. 0 preço comportamental apresentou $\beta=0,290$ e significância $0,027(p<0,05)$ e o preço monetário $\beta=0,550$ e significância 0,001 ( $p<0,05)$. Entende-se que preço está se referindo ao sacrifício no qual o consumidor necessita fazer para adquirir o serviço (PETRICK, 2002; SWEENEY; SOUTAR, 2001; ZEITHAML, 1988) e essa perspectiva diz respeito ao que está sendo comprado (HOLBROOK, 1999). Continuando a observação do perfil dos respondentes, sendo relevante na situação de compra de serviços no mercado fitness. Neste quesito fica evidente que o consumidor do mercado fitness é vulnerável ao preço.

No que diz respeito ao preço comportamental, os alunos/clientes entendem ser mais fácil a contratação, compra e negociação em estabelecimentos FísICOS em comparação ao VIRTUAL, contudo, no que se refere ao preço monetário, os clientes entendem ser mais econômico a loja VIRTUAL em detrimento ao estabelecimento FísICO, embora que, considerem o preço justo, valendo o investimento aplicado.

Nas hipóteses que trataram dos construtos sobre confiança e intenção de compra, a relação da confiança no provedor de serviços ( $H 2)$ apresentou $\beta=0,450$ e significância $0,020(p<0,05)$, suportando a hipótese. Com relação à confiança na empresa, o resultado segue os achados encontrado por outros estudos, tanto no comportamento de compra (McCOLE; RAMSEY; WILLIAMS, 2010), quanto no comportamento de recompra (CURTH et al., 2020; ROHDEN; MATOS; CURTH, 2016). Percebe-se que o 
resultado de confiança do aluno/cliente para os serviços de forma física ainda supera o formato virtual, quando comparados. Nesse caso, entende-se ser positivo para as academias de ginástica com serviços no formato FÍSICO, pois o resultado ressalta que o aluno/cliente se sente mais seguro estando na presença de um profissional de Educação Física, tendo a disposição sua assistência, assim como também se sente seguro tendo a sua privacidade preservada no momento de compra, facilitando desta forma a intenção de compra.

Por fim, a relação que tratou da hipótese sobre a necessidade de interação e a intenção de compra foi positivamente comprovada, apresentando $\beta=0,60$ e significância $0,001 \quad(p<0,05)$. A necessidade de interação possui o maior coeficiente, indicando a importância que os consumidores ainda atribuem ao contato com o professor/instrutor e funcionários da empresa provedora. Por mais que a internet proporcione uma infinidade de informações sobre produtos e profissionais, a pesquisa e a filtragem de todo o conteúdo disponivel consomem tempo e pode gerar insegurança no consumidor. Neste sentido, o estabelecimento físico apresenta um importante elemento que lhe proporciona vantagem competitiva, desde que bem gerenciado.

Esta dimensão está relacionada à qualidade do relacionamento entre consumidores e empresas, visto que, a interação acontece o tempo inteiro, sendo uma das formas de ligação entre produto/serviço e o consumidor, o que é também chamado de "facilitadores", como é apresentado no estudo de Goudge et al. (2017). Ao compreender esta ligação, é possivel se posicionar melhor as necessidades do consumidor. Este é um fator relevante para academias de ginástica com serviços no formato FíSICO, que tem a disposição do profissional de Educação Física para interagir, facilitar, auxiliar e acompanhar o aluno/cliente durante o seu treino, proporcionando maior segurança e criando afinidades profissionais, pois as pesquisas mostram que relacionamentos fortes com clientes tendem a aumentar sua lealdade e divulgação, gerando lucros corporativos mais altos (SÁNCHEZ et al., 2007). Nas palavras de Petrick (2002), as empresas podem se beneficiar, atraindo consumidores mais propensos à compra e possivelmente mais leais.

\section{CONSIDERAÇÕES FINAIS}

Com o avanço da pandemia decorrente do vírus SARS CoV-2 (COVID-19), grandes empresas fecharam no Brasil e outras tantas se viram obrigadas a se reinventar. No campo da Educação Física não foi diferente. Com o isolamento social, muitas academias de ginástica optaram por oferecer seus produtos/serviços de forma remota e outros aderiram às vendas on-line. Neste contexto, percebe-se, contudo, que muitos empreendedores ainda não têm clareza sobre sua modalidade de atuação e sentem dificuldades em dar segmento a novas propostas de negócios, principalmente no ambiente virtual. 
Neste contexto, o presente estudo teve como foco a intenção de compra de forma comparativa entre os ambientes físicos do mercado fitness versus o ambiente on-line. Dentre diversas variáveis que podem afetar a intenção de compra, este estudo analisou a influência da percepção de valor, confiança e necessidade de interação. Houve esse foco pois esses são fatores que o ambiente de entrega de serviços fitness altera ou pode alterar a avaliação do consumidor.

Os resultados sugerem que o consumidor de serviços do mercado fitness valoriza a interação humana, é sensivel a preço, sente-se mais confortável em adquirir produtos de lojas físicas e valoriza a confiança na empresa para sua decisão de compra. Com relação à comparação entre lojas físicas e virtuais, também foi evidenciado que a resposta emocional é mais relevante no ambiente físico.

Com base nisso, pode-se concluir que os consumidores ainda estão preferindo os ambientes físicos de entrega de serviços fitness. Porém, a experiência vivida na pandemia e a tendência a partir dela é de fortalecimento também dos ambientes virtuais. Logo, ampliar a confiança do consumidor na sua plataforma ou mecanismo de entrega de serviços on-line é mandatório.

Entender as pistas que os consumidores usam para perceber o valor no ambiente físico do mercado fitness e como transportar essas pistas para o ambiente on-line a fim de manter a mesma percepção de valor também é necessário do ponto de vista gerencial.

Também é interessante observar que muitas academias de ginástica perdem oportunidades por não desenvolverem adequadamente relacionamento com seus clientes, além de não possuir programas de retenção de clientes bem estruturados. A maior parte das empresas deste segmento foca apenas na atração de novos clientes. Ao fazer isso, as academias de ginástica aumentam a necessidade de ações promocionais, aguçam a sensibilidade ao preço de mercado e enfraquecem o valor percebido pelos consumidores com relação ao serviço que elas efetivamente prestam. Além disso, ao deixarem de manter contato ativo com seus clientes, as academias de ginástica perdem a oportunidade de minimizar o impacto de eventuais expectativas não atendidas de seus clientes, de planejar interações futuras e de estimular a intenção de compra de seus melhores clientes.

A satisfação das necessidades dos clientes implica atenção constante a estes, a fim de estabelecer relações duradouras (e não apenas durante a transação) e de confiança entre a empresa e os potenciais alunos/clientes, visto que uma das principais reclamações dos alunos de academias de ginásticas com estabelecimento físico é o sentimento de "abandono", uma falta de política de pós-venda, dando ênfase em apenas captar clientes, deixando de dar suporte aos clientes já conquistados a mais tempo. Já no ambiente virtual, o principal obstáculo é a falta de confiança que antecede a compra. Portanto, é importante que as academias de ginástica identifiquem adequadamente os segmentos que poderão atender melhor de acordo com suas competências corporativas e recursos disponíveis. 
Por último, é pertinente comentar que novas pesquisas sobre os antecedentes da intenção de compra podem verificar a influência de outras características do consumidor. Aspectos como faixa etária, renda e perfil do cliente (sozinho, casado, com crianças pequenas, grau de escolaridade etc.) podem alterar o perfil de compra, a facilidade de uso de tecnologia, a propensão ao risco de imprevistos, resposta emocional e confiança. Aprofundar a compreensão sobre a resposta emocional do consumidor é uma variável que merece atenção, avaliar se ela é influenciada por alguma característica do consumidor e quais fatores são mais relevantes em lojas físicas e em ambientes virtuais pode evidenciar as principais características comerciais do setor.

\section{REFERÊNCIAS}

BA, S.; PAVLOU, P. A. Evidence of the Effect of Trust Building Technology in Electronic Markets: Price Premiums and Buyer Behavior. MIS Quarterly, Minneapolis, v. 26, n. 3, p. 243-268, 2002. DOI: $10.2307 / 4132332$.

BAGOZZI, R. P. A Holistic methodology for modeling consumer response to innovation. Operations Research, Catonsville, v. 31, n.1, p. 128-176, 1983. DOI: 10.1287/opre.31.1.128.

BAUMEISTER, R. F.; LEARY, M. R. The need to belong: desire for interpersonal attachments as a fundamental human motivation. Psychological Bulletin, Washington, v. 117, n. 3, p. 497-529, 1995. DOI: 10.1037/0033-2909.117.3.497.

CHEN, Y.-H.; BARNES, S. Initial trust and on-line buyer behaviour. Industrial Management \& Data Systems, [s. I.], v. 107, n. 1, p. 21-36, 2007. DOl: 10.1108/02635570710719034.

COLLIER, J. E.; KIMES, S. E. Only if it is convenient: understanding how convenience influences self-service technology evaluation. Journal of Service Research, [s. I.], v. 16, n. 1, p. 39-51, 2013. DOI: $10.1177 / 1094670512458454$.

CURTH, M.; PICOTO, W. N.; GARCIA. A. S.; NETO, A. F. G. Trust in online shopping: a comparative study between Brazil and Portugal. Brazilian Journal of Marketing, Vitória, v. 19, n. 2, p. 237-260, 2020. DOl: 10.5585/remark.v19i2.15566.

DAVIS, F. D.; BAGOZZI, R. P.; WARSHAW, P. R. User acceptance of computer technology: a comparison of two theoretical models. Management Science, [s. I.], v. 35, n. 8, p. 982-1003, 1989. DOI: 10.1287/ mnsc.35.8.982. 
DE RUYTER, K.; WETZELS, M.; LEMMINK, J.; MATTSON, J. The dynamics of the service delivery process: a value-based approach. International Journal of Research in Marketing, Amsterdã, v. 14, n. 3, p. 231 243, 1997. DOI: 10.1016/S0167-8116(97)00004-9.

DEHESHTI, M.; FIROUZJAH, J. A.; ALIMOHAMMADI, H. The relationship between brand image and brand trust in sporting goods consumers. Annals of Applied Sport Science, [s. I.], v. 4, n. 3, p. 27-34, 2016. DOI: 10.18869/acadpub.aassjournal.4.3.27.

DELIBERALI, E. T.; BRANDAO, M. M.; BIZARRIAS, F. S. Repurchase intention conditioned to economic confidence in Main Street Retail Districts. Brazilian Businesss Review, Vitória, v. 16, n. 6, p. 589-606, 2019. DOI: 10.15728/bbr.2019.16.6.4.

DEMIRCI OREL, F; KARA, A. Supermarket self-checkout service quality, customer satisfaction, and loyalty: empirical evidence from an emerging market. Journal of Retailing and Consumer Services, Londres, v. 21, n. 2, p. 118-129, 2014. DOI: 10.1016/j.jretconser.2013.07.002.

EISENBEISS, M.; CORNELIBEN, M.; BACKHAUSS, K.; HOYER, W. D. Non linear and asymmetric returns on customer satisfaction: do they vary across situations and consumers? Journal of Academy Marketing Science, Greenvale, v. 42, p. 242-263, 2014. DOI: 10.1007/s11747-013-0366-1.

EISINGERICH, A. B.; BELL, S. J. Perceived service quality and customer trust: does enhancing customers' service knowledge matter? Journal of Service Research, [s. I.], v. 10, n 3, p. 256-268, 2008. DOI: $10.1177 / 1094670507310769$.

GARRÁN, V. G. De navegadores a compradores: os elementos motivacionais nas decisões de compra pela Internet. Contextus - Revista Contemporânea de Economia e Gestão, Fortaleza, v. 3, n. 1, p. 45 54, 2005. DOI: 10.19094/contextus.v3i1.32063.

GARSON, G. D. Structural equation modelling. Asheboro: Statistical Associates Publishers, 2012. 227 p.

GONÇALVES, C. S.; COSTA, L. M.; MAIA, C. R. Atuação de influenciadores digitais: relação entre credibilidade e intenção de compra dos novos consumidores. SINERGIA - Revista do Instituto de Ciências Econômicas, Administrativas e Contábeis (ICEAC), Rio Grande, v. 23, n. 2, p. 21-31, 2019. DOI: 10.17648/ sinergia-2236-7608-v23n2-8229.

GOUDGE, D.; GOOD, M. C.; HYMAN, M. R.; AGUIRRE, G. Modeling specialty store customers' buy/no-buy decisions. International Journal of Retail \& Distribution Management, Bingley, v. 45, n. 12, p. $1260-$ 1276, 2017. DOI: 10.1108/IJRDM-03-2017-0036. 
GRÖNROOS, C.; HEINONEN, F.; ISONIEMI, K.; LINDHOLM, M. The NetOffer model: a case example from the virtual marketspace. Management Decision, Londres, v. 38, n. 4, p. 243-252, 2000. DOI: 10.1108/00251740010326252.

GROTH, J. C. (1995). Exclusive value and the pricing of services. Management Decision, Londres, v. 33, n. 8, p. 22-29. DOI: 10.1108/00251749510093905.

HAIR JUNIOR, J. F.; BLACK, W. C.; BABIN, B. J.; ANDERSON, R. E.; TATHAM, R. L. Análise multivariada de dados. 6. ed. Porto Alegre: Bookman, 2009. 688 p.

HELLIER, Phillip K. et al. Customer repurchase intention: A general structural equation model. European journal of marketing, 2003.

HOLBROOK, M. B. Consumer value: a framework for analysis and research. Londres: Routledge, 1999. $224 \mathrm{p}$.

KIM, D. J.; FERRIN, D. L.; RAO, H. R. A trust-based consumer decision-making model in electronic commerce: the role of trust, perceived risk, and their antecedents. Decision Support Systems, Amsterdã, v. 44, n. 2, p. 544-564, 2008. DOI: 10.1016/j.dss.2007.07.001.

$L E E, H$. J. Personality determinants of need for interaction with a retail employee and its impact on self-service technology (SST) usage intentions. Journal of Research in Interactive Marketing, Londres, v. 11, n. 3, p. 214-231, 2017. DOI: 10.1108/JRIM-04-2016-0036.

LEE, I. M.; SHIROMA, E. J.; LOBELO, F.; PUSKA, P.; BLAIR, S. N.; KATZMARZYK, P. T. Effect of physical inactivity on major non-communicable diseases worldwide: an analysis of burden of disease and life expectancy. The Lancet, Londres, v. 380, n. 9838, p. 219-29, 2012. DOI: 10.1016/S0140-6736(12)61031-9. PMID: 22818936. PMCID: PMC3645500.

MCCOLE, P.; RAMSEY, E.; WILLIAMS, J. Trust considerations on attitudes towards online purchasing: The moderating effect of privacy and security concerns. Journal of Business Research, Amsterdã, v. 63, n. 9/10, p. 1018-1024, 2010. DOI: 10.1016/j.jbusres.2009.02.025.

MCKNIGHT, D. H.; CHOUDHURY, V.; KACMAR, C. The impact of initial consumer trust on intentions to transact with a web site: a trust building model. The Journal of Strategic Information Systems, Amsterdã, v. 11, n. 3-4, p. 297-323, 2002. DOI: 10.1016/S0963-8687(02)00020-3.

MEUTER, M. L.; OSTROM, A. L.; BITNER, M. J.; ROUNDTREE, R. The influence of technology anxiety on consumer use and experiences with self-service technologies. Journal of Business Research, Amsterdã, v. 56, n. 11, p. 899-906, 2003. DOI: 10.1016/S0148-2963(01)00276-4. 
MEUTER, M. L.; OSTROM, A. L.; ROUNDTREE, R. I.; BITNER, M. J. Self-service technologies: understanding customer satisfaction with technology-based service encounters. Journal of Marketing, Los Angeles, v. 64, n. 3, p. 50-64, 2000. DOI: 10.1509/jmkg.64.3.50.18024.

MIYAZAKI, A. D.; FERNANDEZ, A. Consumer perceptions of privacy and security risks for online shopping. Journal of Consumer Affairs, [s. I.], v. 35, n. 1, p. 27-44, 2001. DOl: 10.1111/j.1745-6606.2001. tb00101.x.

MOON, J.; CHADEE, D.; TIKOO, S. Culture, product type, and price influences on consumer purchase intention to buy personalized products online. Journal of Business Research, Amsterdã, v. 61, n. 1, p. 31-39, 2008. DOI: 10.1016/j.jbusres.2006.05.012.

MOREIRA, J. C. T. (coord.). Administração de vendas. 2. ed. São Paulo: Saraiva. 2007. 408 p.

OLIVER, R. L. Satisfaction: a behavioral perspective on the consumer. 2. ed. Nova lorque: Routledge, 2010. 544 p.

PEÑA-GARCÍA, Nathalie et al. Purchase intention and purchase behavior online: A cross-cultural approach. Heliyon, v. 6, n. 6, p. e04284, 2020.

PETRICK, J. F. Development of a multi-dimensional scale for measuring the perceived value of a service. Journal of Leisure Research, Londres, v. 34, n. 2, p. 119, 2002. DOI:

10.1080/00222216.2002.11949965

ROHDEN, S. F.; MATOS, C. A.; CURTH, M. Intenções de recompra e confiança do consumidor on-line. Revista de Ciências da Administração, Florianópolis, v. 18, n. 45, p. 9-24, 2016. DOI: 10.5007/2175-8077.2016v18n45p9.

RUBIN, A. M.; PERSE, E. M.; POWELL, R. A. Loneliness, parasocial interaction, and local television news viewing. Human Communication Research, Londres, v. 12, n. 2, p. 155-180, 1985. DOI: 10.1111/ j.1468-2958.1985.tb00071.x.

RUST, R. T.; ESPINOZA, F. How technology advances influence business research and marketing strategy. Journal of Business Research, Amsterdã, v. 59, n. 10-11, p. 1072-1078, 2006. DOI: 10.1016/j.jbusres.2006.08.002.

SÁNCHEZ, J.; CALLARISA, L.; RODRÍGUEZ, R. M.; MOLINER, M. A. Perceived value of the purchase of a tourism product. Tourism Management, Londres, v. 27, n. 3, p. 394-409, 2006. DOI: 10.1016/j.tourman.2004.11.007. 
SCHAUPP, L. C.; BÉLANGER, F. A conjoint analysis of online consumer satisfaction. Journal of Electronic Commerce Research, [s. I.], v. 6, n. 2, p. 95-111, 2005.

SHETH, J. N.; NEWMAN, B. I.; GROSS, B. L. Consumption values and market choice. Cincinnati: South Western Publishing, 1991. 218 p.

STEENKAMP, J. B. E.; MAYDEU-OLIVARES, A. Stability and change in consumer traits: evidence from a 12-year longitudinal study, 2002-2013. Journal of Marketing Research, Londres, v. 52, n. 3, p. 287308, 2015. DOI:10.1509/jmr.13.0592.

SWEENEY, J. C.; SOUTAR, G. N. Consumer perceived value: The development of a multiple item scale. Journal of Retailing, Nova lorque, v. 77, n. 2, p. 203-220, 2001. DOI: 10.1016/50022-4359(01)00041-0.

SWEENEY, J. C.; SOUTAR, G. N.; JOHNSON, L. The role of perceived risk in the quality-value relationship: a study in a retail environment. Journal of Retailing, Nova lorque, v. 75, n. 1, p. 77-105, 1999. DOI: 10.1016/S0022-4359(99)80005-0.

TAYLOR, S. A.; BAKER, T. L. Uma avaliação da relação entre a qualidade do serviço e a satisfação do cliente na formação das intenções de compra dos consumidores. Journal of Retailing, Nova lorque, v. 70, n. 2, p. 163-178, 1994. DOI: 10.1016/0022-4359(94)90013-2.

TERZIA, N. The impact of e-commerce on international trade and employment. Procedia Social and Behavioral Sciences, Moscou, v. 24, p. 745-753, 2011. DOI: 10.1016/j.sbspro.2011.09.010.

WALKER, R. H.; CRAIG-LEES, M.; HECKER, R.; FRANCIS, H. Technology-enabled service delivery: An investigation of reasons affecting customer adoption and rejection. International Journal of Service Industry Management, Londres, v. 13, n. 1, p. 91-106, 2002. DOI: 10.1108/09564230210421173.

WEISBERG, J.; TE'ENI, D.; ARMAN, L. Past purchase and intention to purchase in e commerce: The mediation of social presence and trust. Internet Research, v. 21, n.1, p. 82-96, 2011. https://doi. org/10.1108/10662241111104893

WELLS, J.; VALACICH, J.; HESS, T. What signal are you sending? How website quality influences perceptions of product quality and purchase intentions. MIS Quarterly, Minneapolis, v. 35, n. 2, p. 373-396, 2011. DOI:10.2307/23044048.

WILSON, S. G.; ABEL, I. So you want to get involved in e-commerce. Industrial Marketing Management, [s. I.], v. 31, n. 2, p. 85-94, 2002. DOI: 10.1016/S0019-8501(01)00188-2. 


\section{Gestãoe \\ Desenvolvimento}

e-ISSN: 2446-6875

p-ISSN: $1807-5436$

WORLD HEALTH ORGANIZATION (WHO). World report on disability. Genebra: World Health Organization, c2011. E-book. Disponivel em: https://www.ncbi.nlm.nih.gov/books/NBK304079/. Acesso em: 10 abr. 2021.

ZEITHAML, V. A. Consumer perceptions of price, quality, and value: a means-end model and synthesis of evidence. Journal of Marketing, Los Angeles, v. 52, n. 3, p. 2-22, 1988. DOI: 10.2307/1251446.

ZEITHAML, V. A.; BITNER, M. J. Marketing de serviços: a empresa com foco no cliente. 2. ed. Porto Alegre: Bookman, 2003. 